\title{
Runaway electron generation in tokamak disruptions
}

\author{
H M Smith ${ }^{1}$, T Fehér ${ }^{1}$, T Fülöp ${ }^{2}$, K Gál $^{3}$, E Verwichte ${ }^{4}$ \\ ${ }^{1}$ Max-Planck-Institut für Plasmaphysik, Greifswald, Germany \\ ${ }^{2}$ Department of Radio and Space Science, Chalmers University of Technology and \\ Euratom-VR Association, Göteborg, Sweden \\ ${ }^{3}$ KFKI RMKI, Association EURATOM, Budapest, Hungary \\ ${ }^{4}$ Centre for Fusion, Space and Astrophysics, University of Warwick, Coventry, UK \\ E-mail: hakan.smith@ipp.mpg.de
}

\begin{abstract}
Runaway electrons can be generated in disruptions by the Dreicer, hot tail and avalanche mechanisms. Analytical and numerical results for hot tail runaway generation are included in a 1-dimensional model of electric field, temperature and runaway current, which is applied to simulate disruptions and fast shutdown. The peaked shape of the runaway current density profile may cause tearing modes to become unstable. Fast shutdown is studied by prescribing varying amounts of injected impurities. Large argon content suppresses runaways in JET simulations but causes hot tail generation in ITER. A pellet code is coupled to the runaway model, and it is extended to enable simulations of carbon doped deuterium pellet injection. Such pellets are seen not to give enough cooling for a fast current quench.
\end{abstract}

\section{Introduction}

The runaway electrons produced by high electric fields in tokamak disruptions may cause severe damage to the vessel wall upon impact. This poses a potential threat to the operation of tokamaks with large currents, such as ITER. The three main runaway generation processes are the Dreicer [1], the hot tail and the avalanche [2] mechanisms, of which the first two generate a small seed population that becomes amplified by the avalanche. We will focus on the hot tail mechanism, which is caused by incomplete thermalization of the electron velocity distribution during the cooling phase of the disruption. All three runaway generation mechanisms are included in a self-consistent 1D model of the the electric field evolution via the induction equation. It is found that the runaway generation causes a peaking of the radial current density profile, which can trigger tearing modes that may in turn affect the current quench time. Runaway electron losses (e.g. due to plasma instabilities [3] or magnetic field fluctuations [4-6]) are not included in this study, which therefore considers worst case scenarios for the runaway generation.

To mitigate the large mechanical and thermal vessel loads in disruptions one may preemptively inject impurities, e.g. using "killer" pellets, to radiatively cool the plasma and safely shut down the discharge. However, this can also lead to runaway electron 
production. Simulations with different prescribed amounts of impurities have been performed using the 1D runaway code, and carbon doped pellet injection scenarios have been studied using a pellet ablation code coupled to the runaway code. The results indicate that deuterium pellets doped with high $Z$ materials, such as argon or neon, are needed in order to both suppress runaways and obtain a sufficiently short current quench time to reduce the mechanical forces on the vessel.

\section{Runaway generation mechanisms}

At suprathermal speeds the dynamical friction force on an electron decreases with increasing velocity. The force from an electric field (e.g. the electric field $E$ induced in a tokamak disruption) therefore dominates over friction above a critical velocity $v_{\mathrm{c}}=v_{T} \sqrt{E_{\mathrm{D}} / 2 E}$, where $v_{T}$ is the thermal velocity, $E_{\mathrm{D}}=\nu m_{\mathrm{e}}^{2} c^{3} / e T$ the Dreicer field, $\nu=n_{\mathrm{e}} e^{4} \ln \Lambda / 4 \pi \epsilon_{0}^{2} m_{\mathrm{e}}^{2} c^{3}$ the relativistic electron collision frequency, $n_{\mathrm{e}}$ the electron density, $T$ the electron temperature and $\ln \Lambda$ the Coulomb logarithm. A runaway generation mechanism is a process which moves electrons into the runaway region $v>v_{\mathrm{c}}$ of velocity space where they are accelerated to become highly energetic runaway electrons. The acceleration can only occur if $E$ is greater than the critical electric field $E_{\mathrm{c}}=m_{\mathrm{e}} c \nu / e$, corresponding to a minimum in the friction force at relativistic velocities. In tokamaks with large current the dominant runaway generation mechanism is the avalanche, which is caused by close range collisions between existing runaways and thermal electrons [2]. The avalanche is a secondary process, for which the necessary initial seed of runaways can be created by several different primary mechanisms. One such process is the Dreicer runaway mechanism, in which electrons diffuse into the runaway region due to a random walk in velocity space caused by long range collisions [1]. In the following we will limit the discussion to the Dreicer and the hot tail runaway electron mechanisms. However, in ITER, tritium decay and Compton scattering of $\gamma$ rays emitted by the activated wall are also possible primary runaway electron sources. Losses of runaways due to magnetic perturbations are important in disruptions, and the dependence on the fluctuation level $\delta B / B$ was investigated in [5]. Here, we for simplicity assume the unknown parameter $\delta B / B$ to be zero to study a worst case scenario without losses, an approach that might be more relevant for impurity injection scenarios than for natural disruptions.

In the rapid thermal quench phase $\left(\tau_{\mathrm{TQ}} \sim 1 \mathrm{~ms}\right)$ of a disruption the electron velocity distribution is not in a steady-state, which is assumed in the derivation of the Dreicer runaway rate [1]. At high velocities the collision frequency is lower than the cooling rate, so in an initial transient phase high energy electrons do not have time to thermalize. They are left as a hot tail, while the low energy bulk of the distribution function cools down, following a Maxwellian with a decreasing temperature $T(t)$. If the cooling is rapid compared with the collision frequency at the runaway threshold velocity, then the hot tail makes the number of electrons in the runaway region higher than what it would be for a Maxwellian with temperature $T(t)$. Many more runaways are therefore produced 
than given by the Dreicer rate. Hot tail runaway generation also differs from Dreicer generation because it is limited in time to the cooling phase, whereas Dreicer generation continues as long as the electric field is high.

The hot tail mechanism was studied numerically in [5,7] and analytically in [8-10]. The analytic work considers thermal quench types where the cooling is caused by an inflow of impurities, e.g. wall material or injected pellets. This mainly cools down the thermal electrons through excitation and ionization processes, whereas the suprathermal electrons slow down due to collisions with the thermal electrons. The number of runaways is approximated by first solving the kinetic equation to determine the evolution of the distribution function $f$ without the influence of the electric field. The obtained velocity distribution is then integrated over the runaway region set up by the electric field. The kinetic equation is

$$
\frac{\partial f}{\partial t}=\frac{\nu c^{3}}{v^{2}} \frac{\partial}{\partial v} 2 G\left(\frac{v}{v_{T}}\right)\left(\frac{v^{2}}{v_{T}^{2}} f+\frac{v}{2} \frac{\partial f}{\partial v}\right)
$$

where $G(x)$ is the Chandrasekhar function, $v_{T}(t)$ is the thermal speed and the temperature $T(t)$ is a specified function of time.

The runaway generation depends sensitively on the final temperature and on the cooling history $T(t)[5,7,9]$. In the special case $T(t)=T_{0}\left(1-t / t_{\text {pow }}\right)^{2 / 3}$, for which $\delta \equiv\left(v_{T}^{3} / 2 \nu c^{3}\right) \mathrm{d} \ln T / \mathrm{d} t$ is constant, the distribution at low temperatures approaches a self-similar solution in the variable $x=v / v_{T}$. Integrating the solution over the runaway region gives the runaway population density estimate [9]

$\frac{n_{\mathrm{run}}}{n_{\mathrm{e}}} \simeq \frac{\delta^{-2 / 3}}{\sqrt{3} \pi} \exp \left(-\frac{3}{5} \delta^{-2 / 3}\right)\left[2 \ln \left(\frac{\delta x_{\mathrm{t}}^{3}-1}{\delta x_{\mathrm{c}}^{3}-1}\right)-3\left(1-\frac{x_{\mathrm{c}}^{2}}{x_{\mathrm{t}}^{2}}\right)\right]+\frac{2 \delta^{1 / 3}}{\sqrt{\pi}} \exp \left(-\delta^{-2 / 3}\right)$,

which holds when $\delta^{-1 / 3}<x_{\mathrm{c}}<x_{\mathrm{t}}$, where $x_{\mathrm{c}}=v_{\mathrm{c} 0} T^{1 / 4} / v_{\mathrm{T} 0} T_{0}^{1 / 4}$ and $x_{\mathrm{t}}^{2}=\delta^{-2 / 3} T_{0} / T$.

In the case of an exponential-like temperature decay $T=T_{\text {final }}+\left(T_{0}-\right.$ $\left.T_{\text {final }}\right) \exp \left(-t / t_{\exp }\right)$, an approximate solution using velocity moments of the kinetic equation yields the runaway density [10]

$$
\frac{n_{\text {run }}}{n_{\mathrm{e}}}=\frac{4}{\sqrt{\pi}} \int_{u_{\mathrm{c}}}^{\infty}\left(1-\frac{\left(u_{\mathrm{c}}^{3}-3 \tau\right)^{2 / 3}}{\left(u^{3}-3 \tau\right)^{2 / 3}}\right) e^{-u^{2}} u^{2} \mathrm{~d} u,
$$

where $u^{3}=v^{3} / v_{T 0}^{3}+3 \tau$ and $\tau(t)$ can be calculated by solving a first order ordinary differential equation, which for large $t$ gives the approximate result $\tau(t) \simeq(t-$ $t \exp ) \nu c^{3} / v_{T 0}^{3}$. In the impurity injection simulations in Section 4 , the temperature evolution is not of any of the special types discussed above. To obtain the number of hot tail runaways, a numerical code that solves (1) has therefore been implemented and benchmarked against the analytical expression (3) for exponential-like temperature decay.

\section{Current profile evolution}

The evolution of the radial profile of the current density is governed by runaway electron generation and resistive diffusion of the electric field. A 1D model of these processes in 
cylinder geometry has been developed [10-12], where the runaway density $n_{\text {run }}$ is the sum of the runaway generation from the Dreicer, hot tail and avalanche mechanisms. The current density is the sum of the Ohmic and runaway current densities $j=\sigma E+n_{\text {run }} e c$, where $\sigma$ is the Spitzer conductivity with a neoclassical correction and the runaway electrons are assumed to travel at the speed of light. The electric field is modelled by the parallel component of the induction equation

$$
\frac{1}{r} \frac{\partial}{\partial r} r \frac{\partial E}{\partial r}=\mu_{0} \frac{\partial}{\partial t}\left(\sigma E+n_{\text {run }} e c\right) .
$$

In the thermal quench, the rapid conductivity drop induces a rising electric field which determines the small seed runaway population from Dreicer and hot tail generation. On the longer current quench time scale $\tau_{\mathrm{CQ}} \sim \mu_{0} \sigma / a^{2}$, where $a$ is the plasma minor radius, the current decays because of resistive diffusion. If the resistive diffusion time scale set by the cold post-thermal quench temperature is much longer than the avalanche growth time scale determined by the electric field, then a large fraction of the initial Ohmic current becomes converted to runaway current.

In computer simulations using the $1 \mathrm{D}$ model (4) for JET $[11,12]$ with an assumed post-thermal quench temperature of $10 \mathrm{eV}$ around half the initial Ohmic current is converted to runaway current, and similar conversion ratios have also been observed experimentally. For exponential-like cooling the simulations show that hot tail dominates over Dreicer generation for short thermal quench times $\left(t_{\exp } \lesssim 0.3 \mathrm{~ms}\right)$ in JET. Moreover, hot tail is less sensitive than Dreicer generation to the post-disruption temperature $T_{\text {final }}$, and Dreicer production generally becomes more suppressed than hot tail generation by increased electron density. In the ITER inductive scenario (central temperature $24.8 \mathrm{keV}$, density $10^{20} \mathrm{~m}^{-3}$, current $15 \mathrm{MA}$ ) simulation in figure 1 with $T_{\text {final }}=10 \mathrm{eV}, t_{\exp }=1 \mathrm{~ms}$ and constant electron density in time, hot tail runaway is the dominant seed generation mechanism [10].

In the simulations, the post-disruption current density carried by the runaway electrons has a radial profile which is more peaked in the centre of the plasma than the pre-disruption current density, and this has also been observed experimentally [13]. The evolution of the current profile during the current quench can make the plasma unstable to tearing modes. It has recently been found [14] that the linear properties of the instability depend on the total current density profile, and that the linear growth is the same irrespective of whether the current is carried by thermal electrons or runaways. However, the nonlinear saturation amplitude is different, and the saturated island size is larger in case of a runaway current than for an Ohmic current, at least in simple slab geometry. These results enable us to determine directly from the calculated current profiles which tearing modes that will become unstable, i.e. have $\Delta^{\prime}>0$, where $\Delta^{\prime}$ is the jump in $\Psi^{\prime} / \Psi$ at the resonant $q=m / n$ surface and $\Psi$ is the poloidal magnetic flux function. In the ITER simulation in figure 1 the initial Ohmic current density profile in figure 1a is replaced by a more peaked runaway current density in approximately $0.5 \mathrm{~s}$, but $\Delta^{\prime}$ for the $q=3 / 2$ and $q=2$ surfaces (figure $1 \mathrm{~b}-\mathrm{c}$ ) becomes positive already after around $0.1 \mathrm{~s}$. Note however that in the real ITER geometry $\Delta^{\prime}$ will be different 

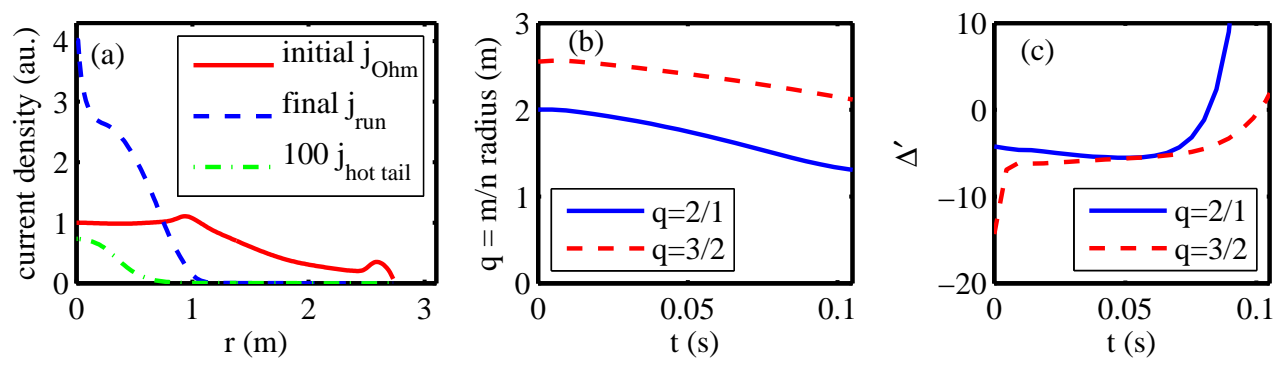

Figure 1. A runaway current simulation for a $15 \mathrm{MA}$ inductive ITER scenario disruption. (a) The pre- and postdisruption current density profiles and the hot tail runaway seed. The latter is multiplied by 100 for better visibility. (b) Position of the resonant surfaces $q=3 / 2$ and $2 / 1$. (c) The evolution of $\Delta^{\prime}$.

from the results obtained here with the 1D cylindrical model. We have not explored the consequences of this instability for the current quench evolution further.

\section{Impurity injection}

To mitigate the large mechanical and thermal vessel loads associated with disruptions it is possible to inject impurities before the onset of the disruption. This can be achieved by injecting gas, liquid jets or "killer" pellets [15-19]. The impurities trigger a fast cooling by radiation, similar to the thermal quench in a disruption. The resulting high resistivity leads to a fast decay of the plasma current, which reduces the mechanical load on the vessel, and since the thermal energy is lost by uniform radiation, the local heat loads on the wall are also reduced. The cooling can result in runaway electron generation, but at the same time the increased electron density due to the injected impurities causes an increase in $E_{\mathrm{c}}$ and $E_{\mathrm{D}}$ which gives a larger threshold for runaway production. However, to completely suppress the runaway production one might need to increase the electron density even more, e.g. by injecting deuterium.

The effects of impurity radiation have been added to the 1D model (4) by including energy balance equations for electrons and ions, taking into account radial heat diffusion, Ohmic heating, line radiation, bremsstrahlung, ionization and collisional energy exchange between different particle species [20]. The time evolution of the charge state populations are described using the so-called rate equations.

To gain an understanding of the physical processes involved, simulations have been performed with prescribed amounts of impurities initially present in the plasma. Figures $2 \mathrm{a}-\mathrm{b}$ show a simulation for a JET-like plasma with $I_{0}=2 \mathrm{MA}, a=1 \mathrm{~m}$, $T_{0}=\left(1-0.9(r / a)^{2}\right) \cdot 4 \mathrm{keV}$ and $n_{\mathrm{D}}=\left(1-0.9(r / a)^{2}\right)^{2 / 3} \cdot 6 \cdot 10^{19} \mathrm{~m}^{-3}$, in which $10 \%$ argon has been added in the beginning of the calculation with the same radial profile as the deuterium density. The introduction of argon atoms causes a rapid radiative collapse, which is however counteracted by increased Ohmic heating due to the rising resistivity. A few localised hot regions form due to a balance between heat diffusion, radiation and Ohmic heating, a phenomenon which was described in [21]. When the 

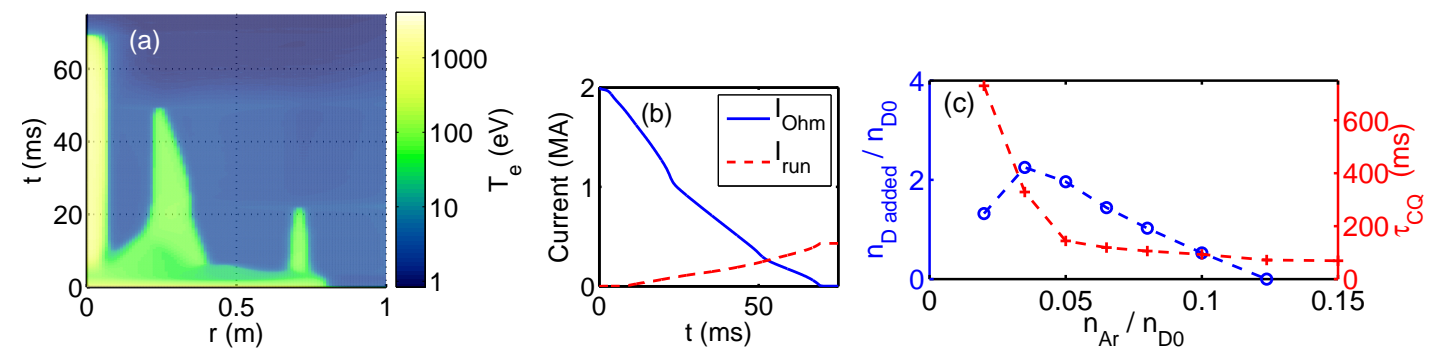

Figure 2. (a) Temperature and (b) currents in a simulation with $10 \%$ argon in JET. (c) A parameter scan for argon injection in JET. Circles (left axis): fraction of extra deuterium required for runaway suppression. Crosses (right axis): resulting current quench time.

hot regions finally disappear their Ohmic current is often partially replaced by a Dreicer and avalanche generated runaway current. In figure 2 a heat diffusion coefficient of $\chi=1 \mathrm{~m}^{2} / \mathrm{s}$ was assumed. A higher $\chi$ generally gives wider, and a different number of hot regions, and the current quench duration and runaway production consequently depend in a non-trivial way on $\chi$.

To suppress the runaway generation that occurs when a high $Z$ material is injected one can simultaneously insert more deuterium in the simulation to raise $E_{\mathrm{D}}$ and $E_{\mathrm{c}}$. The amounts of added argon and deuterium are varied in the JET simulations in figure 2c. As functions of the inserted argon fraction, the crosses show the current quench time, and the circles mark how much additional deuterium is needed to keep the runaway current below $0.1 \%$ of the initial $2 \mathrm{MA}$ Ohmic current. For high argon fractions $(>13 \%)$ no additional deuterium is required because of the large amount of electrons delivered by the argon itself. The trend that a high argon content suppresses runaway generation is also observed in TEXTOR massive gas injection experiments and 0D modelling [19].

ITER simulations for low argon densities show a similar behaviour as the JET cases. A radiative collapse starts at the edge and begins to propagate inwards, see figure $3 \mathrm{a}$. Localised hot regions form, where a Dreicer runaway seed is generated. Greater argon content, as shown in figure $3 \mathrm{~b}$, leads to a rapid thermal quench throughout the plasma and the generation of a hot tail runaway current, which is amplified by the avalanche to a large fraction of the initial current. The runaway suppression for high argon fractions seen in the JET simulations is thus not expected in ITER.

\subsection{Pellet injection}

The prescribed impurity density profiles used in the simulations in the previous section are very difficult to achieve in practice. To be more realistic, the material deposition and cooling caused by injected gas or killer pellets should be calculated. To model killer pellet injection, a pellet ablation code was coupled to the runaway code described above [20]. The pellet code contains a time dependent physical model of the field elongated neutral and ionized cloud surrounding the pellet. It describes the hydrodynamic expansion of the cigar shaped cloud along the field lines using Lagrangian techniques, and includes atomic 

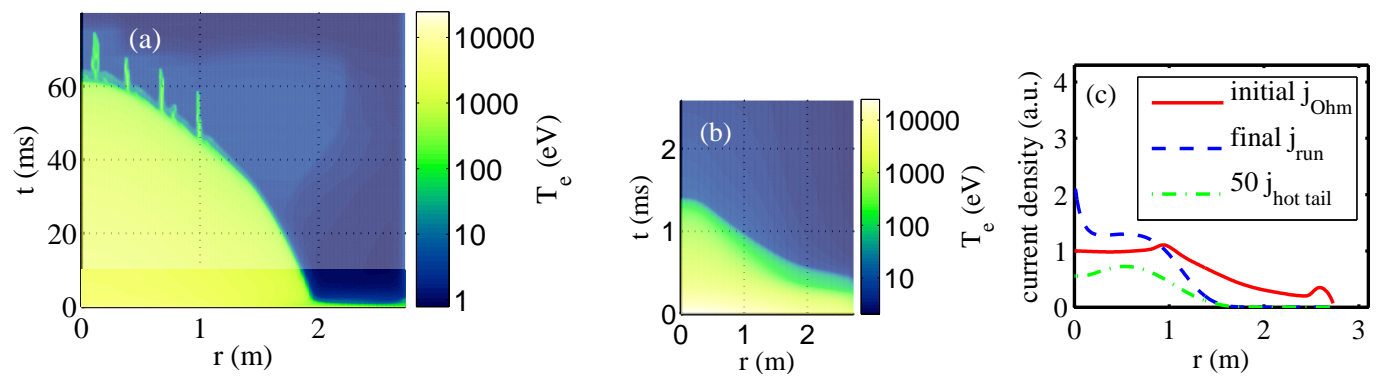

Figure 3. ITER simulations with $\chi=1 \mathrm{~m}^{2} / \mathrm{s}$ where the argon density is increased to its final value in $1 \mathrm{~ms}$. (a) $n_{\mathrm{Ar}} / n_{\mathrm{D}}=0.1$ (resulting in $I_{\text {run }} / I_{0}=0.6$ ) (b) $n_{\mathrm{Ar}} / n_{\mathrm{D}}=1$ $\left(I_{\text {run }} / I_{0}=0.5\right)$ (c) initial, final and hot tail current profiles for the simulation in (b).
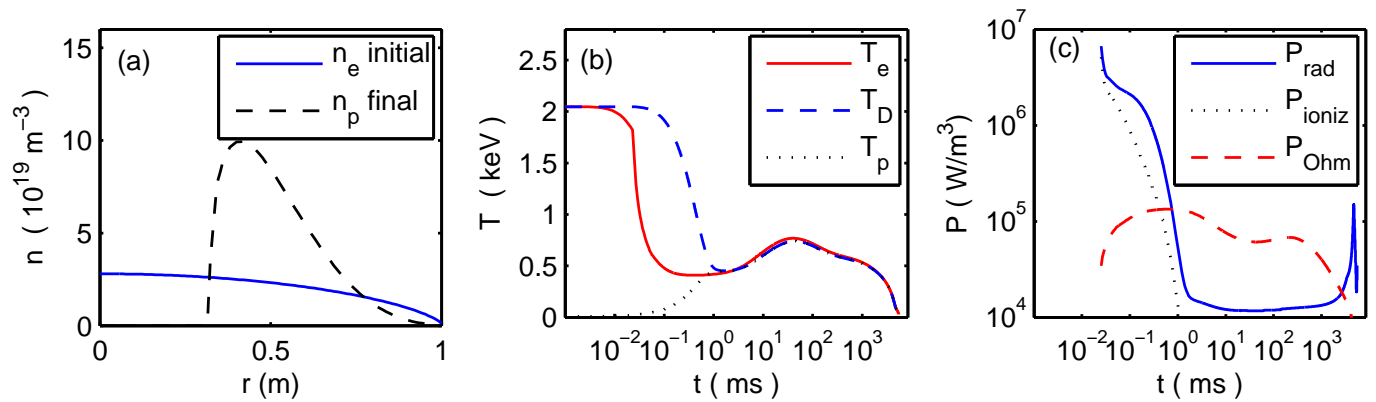

Figure 4. Simulation of a $1000 \mathrm{~m} / \mathrm{s}, 1 \%$ carbon doped deuterium pellet with radius $1.6 \mathrm{~mm}$ in JET. (a) Initial electron density profile $n_{\mathrm{e}}$ and deposited pellet ion profiles $n_{\mathrm{p}}$. (b) Temperature evolution of electrons, deuterium ions and carbon ions at $r / a=0.5$. (c) Radiation, ionization and Ohmic heating power densities at $r / a=0.5$ shown after $0.02 \mathrm{~ms}$ when the data is passed from the pellet to the runaway code.

processes, penetration of ambient plasma particles, heat diffusion into the cloud and the electrostatic shield formation at the periphery. In the future, particle drift effects [22] will also be included. The code calculates the flux surface averaged temperatures and densities of the different particle species during the pellet lifetime, and gives this as input to the runaway code.

The purpose of integrating the pellet and runaway codes is to try to identify pellet injection scenarios that are suitable for disruption mitigation, i.e. give short current quench times and suppress runaway electron generation. As the previous section indicated, it is desirable to inject deuterium and impurity materials simultaneously. Therefore, the code has been extended to deal with impurity doped deuterium pellets. Figure 4 presents a simulation of carbon doped pellet injection. In general, it is found that carbon and carbon doped deuterium pellets give too little cooling. In the simulation in Figure 4 the resulting current quench was several seconds (and no runaways were produced). Therefore, pellets doped with higher $Z$ materials, such as argon or neon is the subject of ongoing studies. Injection of multiple pellets may also be necessary in order to achieve a suitable deposition profile. 


\section{Conclusions}

A 1D model of runaway production and temperature and current density evolution has been used to simulate disruptions and fast shutdown scenarios in JET and ITER. The tearing mode stability of the post-disruption runaway current dominated plasma is modelled and may be important for the current quench dynamics in ITER. A parameter scan has been performed for different amounts of injected argon in JET, and the deuterium density increase required for runaway suppression was determined. When a large amount of pure argon is introduced in JET, runaway generation is suppressed by the high electron density, but in ITER a high argon content leads to hot tail runaway generation. Carbon doped deuterium pellet simulations were performed with a pellet ablation code coupled to the runaway code. Carbon does not provide sufficient cooling, so the pellet code is being developed to enable argon and neon doped pellet simulations.

\section{Acknowledgments}

This work was funded jointly by the European Communities under Association contracts between EURATOM, Hungary, Vetenskapsrådet and Germany and by the United Kingdom Engineering and Physical Sciences Research Council. The views and opinions expressed herein do not necessarily reflect those of the European Commission.

\section{References}

[1] Connor J W and Hastie R J 1975 Nucl. Fusion 15415

[2] Rosenbluth M N and Putvinski S V 1997 Nucl. Fusion 371355

[3] Fülöp T, Smith H M and Pokol G 2009 Phys. Plasmas 16022502

[4] Helander P, Eriksson L G and Andersson F 2000 Phys. Plasmas 74106

[5] Harvey R W, Chan V S, Chiu S C, Evans T E, Rosenbluth M N and Whyte D G 2000 Phys. Plasmas $\mathbf{7} 4590$

[6] Yoshino R and Tokuda S 2000 Nucl. Fusion 401293

[7] Chiu S C, Rosenbluth M N, Harvey R W and Chan V S 1998 Nucl. Fusion 381711

[8] Helander P, Smith H, Fülöp T and Eriksson L G 2004 Phys. Plasmas 115704

[9] Smith H, Helander P, Eriksson L G and Fülöp T 2005 Phys. Plasmas 12122505

[10] Smith H and Verwichte E 2008 Phys. Plasmas 15072502

[11] Eriksson L G, Helander P, Andersson F, Anderson D and Lisak M 2004 Phys. Rev. Lett. 92205004

[12] Smith H, Helander P, Eriksson L G, Anderson D, Lisak M and Andersson F 2006 Phys. Plasmas 13102502

[13] Gill R D, Alper B, Edwards A W, Ingesson L C, Johnson M F and Ward D J 2000 Nucl. Fusion 40163

[14] Helander P, Grasso D, Hastie R J and Perona A 2007 Resistive stability of a plasma with runaway electrons Phys. Plasmas 14122102

[15] Pautasso G, Büchl K, Fuchs J, Gruber O, Herrmann A, Lackner K, Lang P, Mast K, Ulrich M and Zohm H 1996 Nucl. Fusion 361291

[16] Yoshino R, Kondoh T, Neyatani Y, Itami K, Kawano Y and Isei N 1997 Plasma Phys. Control. Fusion 39313

[17] Taylor P L et al 1999 Phys. Plasmas 61872

[18] Hollmann E et al 2008 Nucl. Fusion 48115007 
[19] Bozhenkov S A et al 2008 Plasma Phys. Control. Fusion 50105007

[20] Gál K, Fehér T, Smith H, Fülöp T and Helander P 2008 Plasma Phys. Control. Fusion 50055006

[21] Putvinski S, Fujisawa N, Post D, Putvinskaya N, Rosenbluth M N and Wesley J 1997 J. Nucl. Mater. 241-243 316

[22] Pégourié B 2007 Plasma Phys. Control. Fusion 49 R87 